\title{
DISASTERS AND EXEMPLIFIED VULNERABILITIES IN A CRAMPED PUBLIC HEALTH INFRASTRUCTURE IN INDIA
}

\author{
Baljeet Kaur \\ Tata Institute of Social Sciences, Mumbai \\ Correspondence: kaur.baljeet2195@gmail.com \\ Received: 01 January; Accepted: 25 March; Published: 10 September
}

\begin{abstract}
The varied connotations to the term 'Development' are been channeled through perception. The perception of a political stakeholder differs from that of a rich-businessman, and again, from those who are lesser endowed. There is a pressing need for the government, to identify and maintain checks and balances between exploitative and responsive governance.

The extension of the healthcare sector is an integral part of this holistic growth, while the customer base has largely financed the industry; the obligation on the hand of government needs to increase. The out of pocket spending by patients covers the finances of the sector by $64.2 \%$. (NSSO, 2014 report). The lesser amount of government spending in the healthcare system is a drawback and has effects on the Industry in a negative frame in a large manner, only $28.6 \%$ of Total Health Expenditure is financed by Govt. of India and therefore, calls for the need for better financing mechanisms in the country in the form of insurance schemes and a smoother flow of the already existing policies and frameworks
\end{abstract}

In the debate of private v/s public hospitals, the paper presents reasons that create a barrier on effective utilization of benefits provided, and further constructs the viewpoint that though expensive, private healthcare services provide more assurance to the population in general. The over-crowding of these public institutions in times of epidemics or otherwise, is a self-indication of the dearth of infrastructure and the kind of impacts the interventions has had in terms of alleviating such grievances.

The several debates that I have tried to analyze and interpret include those of the intersections the individuals of the country and the lawmakers have crossed in terms of developmental projects and whether these promises hold true in terms of concrete reality. The depth of understanding and entering these discussions is only a gateway to more pertinent questions of whether the present infrastructure has dwindled due to disasters in the past? Are we actually moving to building resiliency or is it just a mock-up present on paper only?

The paper reflects qualitatively on several government reports on health and the state of the hospitals presented within various contexts of Disasters in the past. The analysis of the National Rural Health Mission, National Urban Health Mission and various others programs initiated by the Government of India and the scope that it has to remove the present day struggled faced by an over-crowded and pressurized public sector healthcare structure.

Key Words: Disasters, Exemplified vulnerabilities, Public Health, Infrastructure 


\section{Introduction}

The ability of a state to provide basic education and healthcare is an important characteristic of a developed nation, as socio-economic parameters have gained vitality in the discussion and discourse on development, and are essential parameters to demarcate the shortcomings and fallouts of a governing regime. In a country that spends only $1.4 \%$ of its GDP on healthcare, it is important to question the vision that the state structure envisages for their population and the healthcare system. The development of a nation is incomplete without considering the progress of all its components and by constantly making an effort to improve the quality of service, ensuring equitability and accessibility to all citizens.

Disasters impede the process of growth, and in some manner, give rise to these perceptive mechanisms. In times of a catastrophe, what might have felt like a milestone in the past may seem as a burden and a liability at present. It is highly likely for income to play a role in determining the parameters for development, i.e. rich countries might enjoy the benefits of spending on a technology that seems like a far-fetched concept for the low-income countries. These conditions thus, predispose certain vulnerabilities to nations with limited resources. Despite of all the challenges that present themselves, there are certain aspects to development that cannot be ignored, primarily, the effective proliferation of education and health sector that constitute a part of the socio-economic component of development. The shift from a purely GDP oriented outlook to growth, to the conception of a rather socially competent nation is an essential turn-around over the years.

There is a growing concern with environment uncertainties, and social vulnerabilities make certain sections susceptible to higher damage than others. Sustainable Development Goals, therefore, focus on eradicating causes of social vulnerability by providing equitable access to basic infrastructure, and thus, a resultant change in the way we perceive development, being an improvement in the holistic human standards, and not just economical gains. The uneven accessibility of resources has been rampant in growing economies, and needs to be altered in order to achieve uniform development. The question therefore, remains of perceptive understanding. In further section, the paper explores the efficiency of private and public healthcare sector, and questions the exploitation of the already vulnerable. Health is a state subject; therefore, center's contribution in improving the conditions has been conflicting, and rather vague. The following paper, therefore, presents arguments from the angle of policy undertaking and the multi-dimensional nature of social development. It deepens the understanding on the existing vulnerabilities, and questions the resiliency of the systems to disasters.

\section{Healthcare Policies in India - Implications and Challenges}

Securing public health and characterization of the declining health statistics in rural India as the need of the hour, drove the resultant shift in policy planning and implementation in the sector. The governmental schemes have been portrayed as being directed at populations that are devoid of basic health benefits and thus aim at enhancing the reach of these policies as well as the quality of healthcare. However, privatization of health system structure, as explored in the further sections, has gained momentum in recent times as private entities are considerably better oriented towards quality and performance based service. In the race of gaining economic benefits, and rising above the competing countries, the ideological shift has become a means to an end approach rather than an all-inclusive endeavor. 
There are several challenges to the approach as it negates the responsibilities of the state and results in inequitable distribution of services on a global index, it has shown some advantages, thus, bypassing the checks that should be considered while constructing such policies.

Understanding the trajectory of expenditure, the health expenditure had declined from $1.3 \%$ of GDP in 1990 to $0.9 \%$ of GDP in 1999. The state-central ratio of health expenditure was 85-15 percent respectively. National Rural Health Mission (NRHM) was launched in 2005 , with a vision to improve the health status of rural India, which is predisposed to vulnerabilities due to lack of accessibility to quality healthcare (Gopalakrishnan \& Immanuel, 2017).

The aim of NRHM was to also make healthcare affordable, effective, accountable, and reliable, with special focus on 18 states (North Eastern States + Empowered action group states [socioeconomically backward states - Bihar, Chhattisgarh, Jharkhand, Madhya Pradesh, Orissa, Rajasthan, Uttarakhand, and Uttar Pradesh] + 2 hilly states (Himachal Pradesh, Jammu \& Kashmir). The National Health Accounts (NHA) 2004-05 data shows that at the State level, $38 \%$ of health expenditure is spend on primary health care, $18.67 \%$ on secondary health care, $21.84 \%$ on tertiary health care and rest on direction and administration and other services. (Gopalakrishnan \& Immanuel , 2017)

Studying the reports by World Health Organization report, (2018) suggests that the situation has changed for better, with number of under 5 deaths reducing from 2049 in 2005 to 1139 (thousand) in 2015, the infant mortality rate has reduced to over the course of 10 years, from $60 \%$ in 2005 to $35.3 \%$ in 2015 , though the number still remains large, and there are aspects that do need attention. The presence of ASHA workers in villages, accredited social health activists does make a difference, and has been observed with the changing statistics over the years of Infant Mortality Rate, Maternal Mortality Rate and Under 5 mortality rate, but the broader question remains, as to whether there is a loophole that is concerning when it comes to providing opportunities. Though, there have been cases where the vulnerabilities of the poor has been high-lightened even in the present scenario, in 2016, Dana Majhi, a tribal from Orissa had to carry his wife from the hospital due to the lack of ambulance, in May 2017, a similar incident occurred in Ettawah, Uttar Pradesh to a man who had to carry his deceased son on his shoulders. The cases are many and the fact that these incidents are encountered is a depiction of the stark reality, which should be enough for us to question the kind of policies that have been implemented, and the emergency of bringing a change, in order to be capable of fulfilling the sustainable developmental goals. (Mishra \& Agarwal, 2017)

The draft National Health Policy 2015 had emphasized, "universal access to good quality health-care services without anyone having to face financial hardship as a consequence"(Dey, 2018). However, the 2017 National Health Policy has maneuvered the control to the private entities, by increasing the fund allocation to them. Thus privatization of health system has become a major focus of the current policy initiatives.

Programs like Janani Suraksha Yojana and Pradhan Mantri Surakshit Matritva Abhiyan (PMSMA) were envisaged to control the increasing maternal mortality rate, though with its implication as well, India could not achieve the Millennium Development Goals (MDGs) significantly in the past. National Urban Health Mission, launched in 2013, a counterpart of NRHM (sub sections of National Health Policy) is coined to require Rs.3, 391crores per year according to the government estimates for it to be effective, though in 2017, the project got an allocation of only Rs.752 crore (Mishra \& Agarwal, 2017).

The inability to achieve MDGs was a mirror to showcase the shortcomings of not implementing holistic approaches in the interventions. The challenges with achieving targets set by these policies remain that of, weak infrastructure and lack of human resources in healthcare services. Primary health centers need to be considered essential and thus, improving and strengthening the PHCs has to be one of the initial targets of the government. 
The lack of skilled personnel providing sufficient and efficient healthcare is and will continue to be a block in eliminating the gaps. The state of public infrastructure is disappointing, and privatization of health system has become a major focus of the current policy initiatives, which has implications of its own, and forms the basis of the arguments explored further in this paper.

\section{Private vs. Public Healthcare}

Despite of the discussion regarding the importance of social parameters that halt the process of development, it has been difficult to eliminate the economic needs of a country, thus the bias towards private sector is prominent, and is fostered by providing the private players a larger space in the health sector. There are factors that prove to be in favor of privatization, as it inculcates the attitude of being result driven. The economic sightedness of the private sector makes them a much better option for the government to put their bets on. The activity of funding private healthcare institutions through various schemes, as described above, is therefore defended by their ability to provide quality, technologically advanced health care services. There are various actors involved in the private sphere of healthcare, including non-profit organizations, which are driven by the need to improve and make efficient healthcare available to all. A study by Basu S, Andrews J, Kishore S, Panjabi R, Stuckler D (2012) has evaluated various secondary sources and have corroborated six factors that influence the argument on public vs. private healthcare options in middle and low-income countries, and describe the preference of one over the other through the lens of a patient. There are multiple factors that affect the choice; the study reviews six themes derived from the WHO framework for health system assessment, including accessibility and responsiveness; quality; outcomes; accountability, transparency and regulation; fairness and equity; and efficiency. The findings are worth to note, as they look at a spectrum of low, middle-income countries. Another study indicates that in 19 of the countries studied, both wealthy and poor families received more care from the private than the public sector, but only when the private sector included private drug shops and similar informal providers (Basu, Andrews, Kishore, Panjabi, \& Stuckler, 2012).

This is indicative of the performance criteria of the private sector providers. The $71^{\text {st }}$ round of NSS on health showcases the dwindling trust in the public health system, as it reports of $58 \%$ rural population and $68 \%$ urban population of preferring private hospitals compared for inpatient care. ${ }^{6}$ Thus, even more patients prefer to be treated in private hospitals, as they trust and feel more secure in the environment, though the study also notes that private institutions do not follow codes and standards, thus also limits their credibility. Being more driven towards, cutting their costs, understaffing and burdening the existing staff is a potential drawback that is prominent in the current scenario of the health sector. Private healthcare sector also runs higher chances to prescribe medicines unnecessarily, as well as suggesting expensive procedures. The exploitation of the patient is impervious and has to be checked in order to increase the efficiency of the private sphere.

In the debate between private and public actors, the ambiguity of what constitutes these actors, also remain a problematic premise, as there are multiple actors, which do not practice legally and are a cause of jeopardy for the vision of creating a sustainable health sector. The accessibility of these services though, is limited, as only few such institutions are affordable and accessible to the weaker sections of the society. As pointed in the previous section, the cases of medical negligence are not fading away, and are significant in highlighting the drawbacks of insufficient funding in the public sector. On the front of equitable services, A World Bank study in Ghana pointed at the lack of evidence noting the difference of user fees in 
public and private sector; however, the data presents the fact that out of pocket expenditure is highest for private not for profits, minimum for public institutions and intermediate for private self financed organizations (Basu, Andrews, Kishore, Panjabi, \& Stuckler, 2012).

Thus, the capacity of the public sector needs to be enhanced looking at the negative factors that make the private sector inaccessible to the vulnerable population. The study also presents the debate on the inclusion of private sector in an efficient manner, though it would also require transparency, which is missing in the present scenario. It is imperative to understand that public private partnerships cannot be biased or directed towards an economic goal, and need to take the perspective of inclusivity. The lack of data to report inefficiencies of programs is a block in forming a PPP that can be successfully implemented. In contemporary times, the debate has taken the form of a competition. The effect of crowding out has resulted in transfer of public funds to private sector, as envisioned by the Health Policy of 2017, India. There are many shortcomings of the public sector, described in the coming section. Though, its ability to provide to a larger population, and being affordable cannot be disputed. On the account of making private sector more affordable, these steps fail to achieve their goals, while also decreasing the funds for the public sector.

Due to better job opportunities in the private sector, physicians too, find it beneficial to run their private practice, and thus, in turn, deepen the exploitation of the patients, by offering their services at a higher rate. Thus, there are always intricacies in the aspect of providing care, and ethics and moral codes are tested, and there needs to be a higher ground in analysing the stand of the medical professionals by bettering prospects in the public sphere as well.

\section{Disasters and Health Infrastructure}

The health infrastructure of India is a little complex to understand within the dynamic state role in the health system structure. It is of little doubt, that there are huge gaps in providing sufficient care to the patients. The dichotomy of public and private sector is in itself unclear, due to lack of data, as mentioned above, though there are clear discrepancies in their functioning.

The Centre's allocation for health has increased from 38cr in 2016-2017 to 47cr in 2017 2018, but the implications of this remain unclear, as it could possibly be directed towards private health systems, which are increasing the burden of demand on public sector from the weaker section. The budget for Pradhan Mantri Swasthya Suraksha Yojana-the Prime Minister's Health Protection Scheme-increased 103\% from Rs 1,953 crore in 2016-17 to Rs 3,975 crore in 2017-18 (D'Cunha, 2017).

As per Rural Health Statistics, 2016 primary and community sub centers are short on human resources by $22 \%$ and $30 \%$ respectively. The understaffing of health centers is an indication on the rise of inadequacies being suffered by those, who do not have the capability to avail care from technologically, advanced hospitals (D'Cunha, 2017).

The cases of Non-communicable and chronic diseases have significantly increased and have taken a turn on the death tolls. Rural health facilities are devoid of basic infrastructure; facilities fall short of water supply, electricity, and connectivity. In case of emergencies, there is a lack of transportation. The statistics are staggering, $63 \%$ of the primary health centers do not have an operation theatre, and while community health centers fall low on specialists, surgeons, gynecologists and pediatricians by $81.5 \%$.

Primary and Secondary care centers are prominently under-staffed and highly insufficient for providing effective solutions, therefore, the tertiary centers are burdened and are 
not able to fulfill the overgrowing demand. These fall short on equipment, and labor to cater to the patients.

There is a growing need for enhancing the human resources, building capacities in order to fulfill the demand and cover all aspects of healthcare. In India, out of the total 1.37 million hospital beds, only 540,000 beds are available in the public hospitals, out of which only $50 \%$ are functional and are concentrated in the top cities. The state of public hospitals in India is poor in terms of infrastructure and more so deficient with respect to the staff. Disasters have the capacity to overwhelm the existing structures, and therefore, the status of the healthcare structure in India is already predisposed to huge economical and structural setbacks in events of a disaster.

The statistics of public and private financing in healthcare all point out towards a lack in quality of services and moreover, a lack of interest in engaging in an efficient manner as a cascading effect of an infrastructural deficiency. The increasing pressure on hospitals to maximize revenues and minimize costs has created a bump in providing efficient and sustained health care to the patients and the situation worsen in times of adverse events (Weismann, et.al, 2007). The Institute of Medicine has defined two major goals in redesigning the system by improving patient safety and enhancing the efficiency, which is been contradicted by the large shortage in demand and supply chain.

Every year, the spread of dengue, chikunguniya, and malaria showcase the inherent inefficiencies of the heath system of the country, and manages to take huge number of lives, due to the inaccessibility and lack of availability of doctors, and space. The question of space is a big one, in an ever-growing economy, notably, second largest we cannot overlook the necessities and adapt to the circumstances, it is essential to grow resiliency, and introspect the implications and the effects of national policies and actions. Despite a history of annual outbreak and spread of vector-borne diseases, these States have not been able to prevent and manage any outbreak.

In the case of earthquakes, floods, and other catastrophes, where the existing hospitals are also affected, and destroyed, the presence of skilled professionals, and an empowered structure is essential in resisting the aftereffects of the disasters. The development of the mental health institutions, and personnel thus also become imperative and a debate that has been rather a part of the long term

Throughout, the various segments of the paper, the emphasis on the interventions in healthcare have been critiqued through the lens of policy operationalization, and farsightedness of paper and reality. Therefore, there is a pressing need to acknowledge the gaps, and work towards creating an equitable and accessible healthcare structure that does not overwhelm the capacities of those involved, as well as complies with the SDGs.

\section{Conclusion}

The paper attempts to review and combine the various dimensions that are important to be considered in order to strengthen the health care system of India. There are multiple factors that influence the smooth functioning of the healthcare facilities, and more often than not; the implications of policy actions determine the nature and efficiency of care. The current dilemma of private vs. public is crucial to understand and contextualize events that demarcate the preference of one over the other. Though, the problems are clearly defined, the solutions are hardly discussed. The widespread advocacy of Public-Private-Partnership is problematic as it results in higher out of pocket expenditure, and thus, contradicting the aim 
with which, the programs have been implemented. The integrated approach to solving such inadequacies is important, as the responsibilities of the state in improving the socio-economic components of the nation has to be the topmost priority as it leads to the generation of a healthy and active service sector. In addition to encountering such situations in times of hazards, the implications of construction more number of AIIMS would not solve the issue at hand, as the existing infrastructure are impaired and need to be improved at a reasonable scale before we can take on more number of institutions. The need to improve data collection, and checks on policy is paramount, as it creates a bigger and better picture for us, to analyze and interpret the lacks of the functioning. The provision of better technology in public institutions thus make an important step in creating a strong and resilient public health system, as it provides better opportunity to the public and helps the vulnerable section of the society rather than burdening them with higher expenditure in a private facility. The component of a competition between the private and public needs to be revisited as the common goal should be welfare. In order to develop economically, one cannot regress socially. Especially, with increasing cases of medical negligence, and disasters that impede the progress, we cannot proceed without working on the following aspects. Epidemics have been on the rise, and with lack of intent on the part of the stakeholders, to expect change is a lost cause; therefore, one needs to revisit the decisions in the context of the changing environmental conditions, and the uncertainty that it brings along with its nature. In any case, no single effort can be recognized until it involves all those affected by the impeding conditions of our healthcare system.

\section{References}

1. Dey, S. (2018, June 20). India's health spend just over 1\% of GDP. Retrieved December 18, 2018, from Times of India: https://timesofindia.indiatimes.com/business/india-business/ indias-health-spend-just-over-1-of-gdp/articleshow/64655804.cms

2. Yadavar, S. (2018, Jan 30). Budget 2018: India's Healthcare Crisis Is Holding back National Potential. Retrieved Dec 19, 2018, from IndiaSpend: https://www.indiaspend.com/ budget-2018-indias-healthcare-crisis-is-holding-back-national-potential-29517/

3. Mishra, P., \& Agarwal, A. (2017, Oct 24). Public Health in India: Gaps in Intent, Policy, and Practice. The Hindu Center for Politics and Public Policy, 30.

4. Gopalakrishnan, S., \& Immanuel , A. (2017, Dec 9). Progress of health care in rural India: a critical review of National Rural Health Mission. International Journal of Community Medicine and Public Health .

5. Basu, S., Andrews, J., Kishore, S., Panjabi, R., \& Stuckler, D. (2012, June 19). Comparative Performance of Private and Public Healthcare Systems in Low- and Middle-Income Countries: A Systematic Review. PLOS Medicine .

6. Bajpai, V. (2014, May 17). The Challenges Confronting Public Hospitals in India, Their Origins, and Possible Solutions . Advances in Public Health .

7. Kumar, A., \& Gupta, S. (2012, July). Health Infrastructure in India: Critical Analysis of Policy Gaps in the Indian Healthcare Delivery. Vivekanand International Foundation .

8. D'Cunha, S. D. (2017, Sep 12). Despite A Booming Economy, India's Public Health System Is Still Failing Its Poor. Retrieved from Forbes: https://www.forbes.com/sites/suparna$\mathrm{dutt} / 2017 / 09 / 12 /$ despite-a-booming-economy-indias-public-health-system-is-still-failing-its-poor/\#40c8b63a78e 0

9. Lokhandwala, Y. (2016). Decline in public health infrastructure in India. Indian Journal of Medical Ethics , 73. 
10. Sharma, K. D. (2012). Implementing Quality Process in Public Sector Hospitals in India: The Journey Begins. Indian Journal of Community Medicine, 150-152.

11. Smith, S. M., Gorski , J., \& vennelakanti, H. (2010). Disaster preparedness and response: a challenge for hospitals in earthquake-prone countries. International Journal of Emergency Management, 209-220. 